University of New Orleans

ScholarWorks@UNO

9-1977

\title{
Single-element rotating-polarizer ellipsometer for film-substrate systems
}

A.-R. M. Zaghloul

R. M.A. Azzam

University of New Orleans, razzam@uno.edu

Follow this and additional works at: https://scholarworks.uno.edu/ee_facpubs

Part of the Electrical and Electronics Commons, and the Physics Commons

\section{Recommended Citation}

A.-R. M. Zaghloul and R. M. A. Azzam, "Single-element rotating-polarizer ellipsometer for film-substrate systems," J. Opt. Soc. Am. 67, 1286-1287 (1977)

This Article is brought to you for free and open access by the Department of Electrical Engineering at ScholarWorks@UNO. It has been accepted for inclusion in Electrical Engineering Faculty Publications by an authorized administrator of ScholarWorks@UNO. For more information, please contact scholarworks@uno.edu. 


\title{
Single-element rotating-polarizer ellipsometer for film-substrate systems
}

\author{
A.-R. M. Zaghloul \\ Electrical Engineering Department, Faculty of Engineering, Cairo University, Cairo, Egypt \\ R. M. A. Azzam \\ Division of Hematology, Department of Internal Medicine, College of Medicine, The University of Nebraska \\ Medical Center, Omaha, Nebraska 68105 \\ and Electrical Materials Laboratory, College of Engineering, University of Nebraska, Lincoln, Nebraska 68508 \\ (Received 18 October 1976; revision received 21 January 1977)

\begin{abstract}
A novel and very simple ellipsometer for the characterization of film-substrate systems that employs one rotating optical element (a polarizer) is proposed. The ellipsometer is based on detecting the angles of incidence at which a film-substrate system has equal amplitude attenuations for light polarized parallel $(p)$ and perpendicular $(s)$ to the plane of incidence. At a certain wavelength, the film thickness of the filmsubstrate system has to lie within permissible-thickness bands (PTB) for the technique to apply.
\end{abstract}

In ellipsometry, a light beam is obliquely reflected from a film-substrate system and its two components parallel $(p)$ and perpendicular $(s)$ to the plane of incidence undergo different amplitude attenuations and phase shifts. By measuring the relative amplitude attenuation $(\tan \psi)$ and the relative phase shift $(\Delta)$ between the $p$ and $s$ components, respectively, we can determine the optical properties of the film and substrate in addition to the film thickness. For a given system, the $p$ and $s$ components may experience the same attenuation at one or more angles of incidence depending on the film thickness. By detecting these angles of incidence at which $\tan \psi=1$, we can fully characterize the filmsubstrate system under consideration if the number of measurements equals the number of parameters to be determined.

Here, we present a novel and simple ellipsometer based on this principle, which has only one element, a polarizer (see Fig. 1). ${ }^{1}$ The polarizer is rotated at a constant angular speed and the angle of incidence is adjusted until the photodetector output is constant (dc). At these angles of incidence, which are the only measurements to be taken, the relative amplitude attenuation is unity. Several such angles of incidence enable the system to be fully characterized.

With a high-quality polarizer, the accuracy of the measurements can be very high because the angle of incidence can be measured accurately. ${ }^{2}$

\section{THE METHOD}

When the linearly polarized light leaving the polarizer is reflected from the sample's surface, the intensity of the light beam is

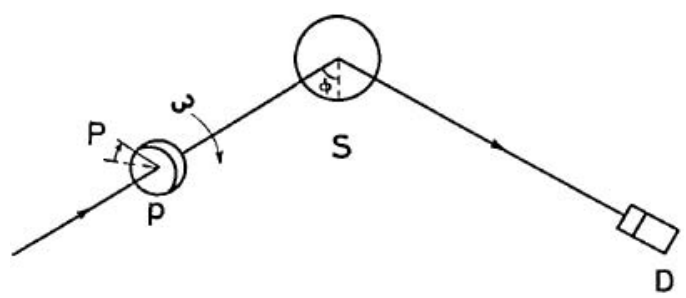

FIG. 1. Single-element rotating-polarizer ellipsometer: $p$, polarizer; $S$, surface; and $D$, photodetector.

$$
I=R_{p} \cos ^{2} P+R_{s} \sin ^{2} P,
$$

where $P$ is the polarizer azimuth measured counterclockwise when looking into the light beam (Fig. 1) and $R_{p}$ and $R_{s}$ are the reflectances for the $p$ and $s$ components of the light beam, respectively. If the polarizer is rotated at a constant angular speed $\omega$, the polarizer azimuth $P$ at a certain time $t$ will be given by $P=\omega t$, and consequently, the reflected light intensity will be

$$
I=R_{p} \cos ^{2} \omega t+R_{s} \sin ^{2} s t .
$$

Therefore, the output of the photodetector $D$ (Fig. 1) will be oscillatory. At the angles of incidence where $\tan \psi=1$, we have $R_{p}=R_{s}\left(\tan ^{2} \psi=R_{p} / R_{s}\right)$, and Eq. (2) becomes

$$
I=R\left(\cos ^{2} \omega t+\sin ^{2} \omega t\right),
$$

or

$$
I=\mathbb{R} \text {, }
$$

where $R=\Re_{p}=R_{s}$. From Eq. (3), it is clear that the intensity of the reflected light beam is constant and does not depend on the polarizer azimuth. Therefore, the photodetector output will be constant (dc).

From the above, we see that the operation of this ellipsometer is very simple. The polarizer is rotated at a constant angular speed and the angle of incidence is adjusted to obtain a dc photodetector output. ${ }^{3}$

This method is applicable to the general case of characterizing absorbing film on absorbing substrate systems. In Ref. 4, there is a clear discussion of the permissible-thickness bands in which the condition of $\tan \psi=1$ exists. Data for the $\mathrm{SiO}_{2}$-Si film-substrate system at a wavelength $\lambda=6328 \AA$, which is applicable to the application of our novel ellipsometer, are presented there. For example, the above system with a film thickness of $1000 \AA$ can be characterized by this ellipsometer (the film thickness lies within a permissiblethickness band). On the other hand, if the film thickness is $2000 \AA$ it cannot be characterized using this ellipsometer at $\lambda=6328 \AA$ (the film thickness lies within a forbidden gap). By changing the wavelength we can move the film thickness into a permissible-thickness band. Any $\mathrm{SiO}_{2}-\mathrm{Si}$ system with a film thickness greater than or equals $5268 \AA$ can be characterized using this ellipsometer and a $6328 \AA$ light source. ${ }^{5}$ 
${ }^{1}$ In 1936, O'Bryan developed a simple ellipsometer that uses a polarizer and a reflector. It depends on detecting the angle of incidence at which $\Delta= \pm \frac{1}{2} \pi$ (principal angle). The angle of incidence and polarizer's azimuth are adjusted to extinguish the reflected beam, see H. M. O'Bryan, "The Optical Constants of Several Metals in Vacuum," J. Opt. Soc. Am. 26, 122 (1936).

${ }^{2}$ The error analysis of this novel ellipsometer, and the use of off null measurements, will be dealt with in full detail in a separate publication.

${ }^{3}$ It is obvious that this ellipsometer can be automated very easily by providing motor drives for the analyzer and angle of incidence.

${ }^{4}$ A. -R. M. Zaghloul, R. M. A. Azzam, and N. M. Bashara, "Design of film-substrate single-reflection retarders," J. Opt. Soc. Am. 65, 1043-1049 (1975).

${ }^{5} \mathrm{SiO}_{2}$-Si film-substrate system having a film thickness as small as $933 \AA$ can be characterized, at $\lambda=6328 \AA$, using this ellipsometer. Using a $2537 \AA$ light source, this novel ellipsometer can be used to characterize the same system having a film thickness as small as $299 \AA$. The shorter the wavelength, the smaller the film thickness. 